\title{
Labyrinthe
}

$37 \mid 2011$ (2)

Des séries et des vies

\section{Appauvrir pour enrichir, une étrange opération philosophique}

Alessandro Delcò

\section{(2) OpenEdition}

1 Journals

Édition électronique

URL : http://journals.openedition.org/labyrinthe/4253

DOI : $10.4000 /$ labyrinthe.4253

ISSN : 1950-6031

Éditeur

Hermann

Édition imprimée

Date de publication : 15 août 2011

Pagination : 149-155

ISBN : 9782705681470

\section{Référence électronique}

Alessandro Delcò, «Appauvrir pour enrichir, une étrange opération philosophique », Labyrinthe [En ligne], 37 | 2011 (2), mis en ligne le 01 août 2013, consulté le 22 avril 2019. URL : http:// journals.openedition.org/labyrinthe/4253; DOI : 10.4000/labyrinthe.4253

Propriété intellectuelle 


\title{
Appauvrir pour enrichir, une étrange opération philosophique
}

\author{
Alessandro Delcò \\ alessandro.delco@univ-paris8.fr
}

Les philosophes aiment et n'aiment pas les faits. C'est que les faits d'un côté amènent la richesse et la diversité du monde, sa phénoménalité exubérante, mais de l'autre entraînent de la confusion. Les faits témoignent de l'irréductible multiplicité du réel, à savoir de ceci, qu'il n'y a rien qui soit d'une seule manière. Ils confortent la thèse suivante: n'existent que des variétés, si bien que le monde se présente en dernière analyse comme une distribution de $\operatorname{cas}^{1}$. Or si chaque chose $-\mathrm{y}$ compris le langage - admet plusieurs façons d'être, variantes souvent très proches les unes des autres, comme telles difficiles à discerner, l'indistinct est nécessairement dans le champ, et avec lui la possibilité du semblant.

Bien qu'ils aient une présence propre incontestable, les faits ne sont pas des blocs d'évidence. Dès qu'on les examine de près, ils montrent toutes sortes de contiguïtés, d'adhérences, d'irrégularités, d'oscillations qui les déforment jusqu'à les obliger parfois à changer de nom (ou à prendre plus d'un nom). L'évidence qui indexe l'effectivité de l'existant n'est jamais apodictique, car hormis les idées pures, rien n'est exact. La plupart du temps on a affaire à des choses aux contours tendanciellement indécis et à la consistance plutôt vague - les variations, ici, loin d'être accidentelles, affectent la structure -, de sorte qu'elles se trouvent occuper l'espace intermédiaire entre elles-mêmes et leur autre en puissance. Il en découle que ce qui est, n'est pas purement et simplement ce qui est. Son identité en règle générale n'est pas une. Sans être tout autre par rapport à ce qu'il est à un instant donné, il supporte des déterminations différentes, dont la suite n'est pas anticipable. Dit autrement: son être changeant en fait un indéterminé relatif déterminable de bien des manières. Sa texture est à même de se modifier dans le temps, sans que l'on sache d'avance si cela va en renforcer ou en affaiblir la teneur propre. Ce qui est sûr,

1. Pascal, Pensées, 479, Paris, Gallimard, Pléiade, t. II, p. 753. 
c'est que cette mutabilité rend sa définition problématique. Il y a peu de cas purs en ce monde ${ }^{2}$.

La philosophie ne peut pas se passer des faits, sous peine de perdre le monde, c'est-à-dire de courir après des entités fantomatiques. En quoi, elle s'enfermerait dans une sphère parfaite mais vide - celle de la nécessité logique -, qui écarte les vérités factuelles sous prétexte qu'elles sont opaques et fortuites. L'ennui, c'est que la philosophie ne peut pas non plus s'y exposer sans précaution, étant donné que dans ce cas elle deviendrait la proie de l'immédiat, où l'illusion niche volontiers. Consentir aux faits jusqu'au bout signifierait en somme pour elle ne plus avoir de recul par rapport au donné, à la contingence, aux passions, dont la force ne peut que l'obnubiler. Dès lors, son souci est d'arriver à voir clair tout en demeurant au plus près de ce qui se manifeste de façon quelque peu aveuglante... Mais cela suppose l'aptitude - très classique, finalement - à rester impassible face à l'événement ${ }^{3}$. Passer à la loupe le visible de première instance sans succomber à la fascination - voilà l'une de ses tâches les plus délicates depuis toujours. Car comment garder sous le regard de l'analyse ce dont l'intensité, en principe, met en échec tout regard? L'œil philosophant peut-il appréhender l'ultime d'un tel apparaître? Est-ce seulement concevable de stabiliser un instant ce qui n'est que passage?

Par définition, un fait ne pourra jamais avoir la transparence de l'idée. D'où la nécessité pour la philosophie de le purifier, d'en éliminer les franges, d'en élider les dimensions accessoires, d'en ôter tous les aspects décoratifs. La soustraction d'existence ${ }^{4}$ est le prix à payer pour pouvoir produire un concept strict de n'importe quelle chose, voire toucher à l'eidos d'un quelconque domaine. Sous ce rapport, la philosophie - depuis Socrate au moins - comporte une dimension d'assèchement inévitable ${ }^{5}$, dans la mesure où elle ne peut avoir lieu qu'en neutralisant une première expression spontanée, à la fois trop chargée, trop positive, et trop conforme (que fait Socrate après tout, sinon entraver le déroulement naturel des activités pour n'en retenir que l'épure schématique?). On ne peut atteindre l'essentiel

2. Kant, Critique de la raison pure, Paris, Gallimard, Pléiade, t. 1, p. 1249.

3. Voir, par exemple, Épictète, Entretiens, II, VI.

4. Bergson, La pensée et le mouvant, Paris, PUF (Bibliothèque de philosophie contemporaine), 1975, p. 157.

5. Platon, Théétète, $150 \mathrm{c}-\mathrm{d}$. 
dans n'importe quelle circonscription du réel sans s'en extraire ${ }^{6}$, voire sans simplifier de façon drastique, c'est-à-dire sans laisser tomber tout ce qui en elle relève de la «parure». Mais pour ce faire, il faut d'abord avoir été au cœur des choses. C'est seulement à condition d'avoir appartenu à un domaine qu'on s'inquiète, si on est philosophe, de ses limites, i.e. des zones de discontinuité par quoi il fait signe vers autre chose que lui. C'est qu'en définitive tout amant de sophia... s'occupe de ce qui dans une chose renvoie en pointillé à son idée. Plus exactement, de toute chose, il essaie de tirer un cas d'idée. Une chose étant donc donnée, il y cherche d'instinct si on peut dire, autre chose - non plus une factualité ponctuelle, chancelante, corruptible, s'accompagnant nécessairement d'une déficience d'être et du cortège de toutes les déceptions qui vont avec, mais le corrélat d'un « $\mathrm{x}$ » impérissable, d'un invisible qui existe plus que ce qu'on a sous les yeux, compte tenu qu'il en est la maximalisation. Soit un tableau par exemple, soit même le plus essentiel des tableaux. L'oeil pensant le trouvera à coup sûr encore trop obéré de fioritures futiles... Non content d'y déceler la répétition d'innombrables autres tableaux sous une forme plus ou moins méconnaissable, il envisagera tous ces tableaux, puis l'ensemble des productions picturales connues à ce jour comme des variations de l'eidos «peinture », variations qui ne l'épuisent en aucune manière. Si remarquables soient-elles, ce sont là de simples réalisations qui, tout en modifiant ce domaine en profondeur, le laissent parfaitement intact en son noyau eidétique. L'idéel seul ne s'use pas. C'est pourquoi il permet de recommencer indéfiniment. En clair, d'autres manières de peindre radicalement différentes sont toujours possibles, contrairement à ce que l'on a tendance à croire. Cela veut dire qu'il y a toujours moyen d'élargir l'idée de peinture, bien que la peinture soit factuellement très fatiguée, étant donné que l'une quelconque de ses versions ${ }^{7} n^{\prime}$ 'est toujours qu'un cas - relativement pauvre - à l'intérieur d'une série ouverte par principe. Mais si, au sein de l'idée, loge ce qui l'excède et qui dans cette mesure ne lui ressemble pas, la peinture - ou la musique ou la poésie ou l'architecture etc. - est toujours toute neuve. Encore une fois, le fait qu'elle ait privilégié telle manière à tel moment

6. N'est-ce pas la quintessence du geste cartésien? Le retranchement vis-à-vis des codes hérités comme préalable à l'institution du philosophique.

7. On n'est pas loin du pluralisme de Nelson Goodman ni de celui, plus essentialiste, de William James. Voir, respectivement, Manières de faire des mondes, trad. M.-D. Popelard, Nîmes, J. Chambon, 1992 (1978), et Philosophie de l'expérience, trad. S. Galetic, Paris, Les Empêcheurs de penser en rond, 2007. 
ne préjuge en rien de son avenir. Même si à chaque fois son expression est complète, il n'en reste pas moins qu'on peut toujours encore la pratiquer tout autrement, si bien qu'elle peut prendre à n'importe quel instant un tour imprévisible. En effet, dès qu'on réussit à la pousser plus loin en intensité, on l'indétermine en lui faisant perdre par surdétermination sa différence propre - perte providentielle, car c'est là que réside très vraisemblablement le secret de l'ouverture infinie du processus créateur.

Les objets philosophiques naissent eux aussi d'une puissance de fiction. Ils prennent corps - un corps très paradoxal, au vrai - grâce à une redite des choses qui les fait disparaître dans leur présence charnelle. Par un mode singulier de la tautologie, le factuel est alors transposé en relation, et du coup investi d'une surexistence. Il se trouve que construire une relation, c'est réussir à se passer de la factualité du monde, de ses vicissitudes empiriques, c'est procurer l'en soi à ce qui par position n'en a pas. L'opérativité philosophique défactualise, et elle défactualise si bien qu'elle fait surgir à elle seule tout un monde - celui des enchaînements démonstratifs - où il n'y a plus de défaillance, de fatigue, de maladie, de mort. Pendant que la pensée s'exerce, le monde est suspendu, le temps mis hors jeu ${ }^{8}$ Alors chaque être est comme irréalisé, et par là même affranchi de toute assignation à des conditions d'existence étriquées. Il est traduit dans un système de relations, et les relations ne meurent pas, à la différence des termes. Du fait de leur simple déploiement, qui est toujours une co-implication, un enrichissement intrinsèque continu se produit. C'est pourquoi la philosophie non seulement chasse la mélancolie, mais rend effectivement éternel - pendant un moment... Toutefois, cette étrange condition d'éternité se paie au prix fort. Elle exige une capacité d'ascèse, à savoir le renoncement à l'hybris jouissive liée à la rencontre du désir d'autrui. Pour arriver à apercevoir des parentés subtiles, il faut congédier les intrigues ordinaires... D'une façon générale, il faut mettre à distance l'effroyable cacophonie d'affects qu'est notre lot quotidien. Ce n'est qu'en faisant taire les voix les plus envahissantes - à côté et en soi-même, à commencer par son propre corps - que la pensée peut se

8. Ce n'est pas tout, et ce n'est pas le plus merveilleux, car le temps suspendu devient source de fécondité. D'une certaine façon, il se résorbe, il se met à couler en lui-même, au lieu de faire œuvre de destruction. Mais en s'auto-alimentant de la sorte, il nourrit puissamment tous les autres processus. Toute chose peut dès lors avoir lieu au-delà d'elle-même, suivant des modalités absolument inattendues, dans une espèce de profusion polymorphe sans exclusive. 
lever. N'empêche que l'inquiétude revient aussitôt, bien que sous d'autres espèces, puisque même l'attitude de réduction ne va pas sans risques. En effet, qui nous garantit qu'à force de soustraire on ne perd pas tout ${ }^{9}$ ?

Non que la philosophie exige qu'on élimine le monde et son propre corps tout court, mais elle nous somme d'en mettre entre parenthèses la stridence, quitte à en exploiter la puissance. C'est qu'elle cherche à pacifier des registres foncièrement incompatibles, tels celui de l'idée et celui du corps. Et elle y arrive presque dans certains cas. Que l'on songe à Merleau-Ponty par exemple, pour qui le corps, loin d'être un étranger radical, est une expérience de densité toute proche de l'idée ${ }^{10}$.

Ce n'est qu'en s'extrayant du grand tintamarre du monde qu'on a quelque chance de pouvoir entendre la fine voix de la philosophie ${ }^{11}$, qui est aussi difficile à discerner, à dire vrai, qu'un accord juste. Le fait est que la justesse des effectuations tient ici à des nuances, de sorte qu'on pourrait être tenté de dire que la philosophie n'est faite au bout du compte que de petits riens. Serait-elle donc insignifiante? Ce n'est pas le mot. Ni fondamentale - trop d'emphase complaisante circule encore dans un énoncé de ce genre - ni dérisoire - il y va tout de même de l'essence des choses, mais d'une essence des plus discrètes -, la philosophie a son importance à côté d'autres activités. Simplement, le grandiloquent n'est pas pour elle $\mathrm{e}^{12}$. À preuve, une figure comme Plotin. Car que nous apprend ce penseur sui generis? Eh bien, il nous apprend à nous débarrasser des idées tristes en convertissant la forme - toute forme - en indice de l'informe ${ }^{13}$. D'après Plotin, on peut toujours neutraliser le sentiment d'être en exil, délaissé, emprisonné dans le fini et le caduc par un regard qui serait à même de voir dans chaque forme s'indiquer un informe potentiellement sans limites. Par conséquent, il s'agit de transférer le visible tout entier en amont, ou pour mieux dire, à l'origine, afin de l'illimiter par le contact - mythique sans doute - avec la source de richesse. En d'autres

9. Chez certains artistes cette démarche peut prendre une tournure dramatique. C'est que par fidélité à l'idée absolue de l'œuvre - son double fantasmatique pur et purement rêvé -, ils cèdent au culte du néant. Patrice Loraux a développé une riche réflexion à ce sujet. Voir notamment les très beaux chapitres qu'il y consacre dans Le tempo de la pensée, Paris, Seuil, 1993, p. 327-360 et 394-420.

10. Même chose dans le fond, malgré des différences capitales, chez Spinoza (dans l'Éthique les attributs se côtoient de très près sans s'agresser).

11. Nietzsche, Ainsi parlait Zarathoustra, Paris, Gallimard, Euvres philosophiques complètes, VI, 1971, p. 151 et Le gai savoir, § 216.

12. Ibidem, § 375 .

13. Ennéades, VI 7, 33. 
termes, il faut détourner le regard de là où il était posé d'abord pour le tourner là où il n'y a rien à voir, rien de subsistant en tout cas, puisque c'est dans cette visée du rien qu'on découvre l'essence de ce qu'on visait, et concomitamment une autre espèce de provenance, qui n'est plus la causalité ontique ordinaire mais l'éclosion de l'être en train de se dissiper. Dès lors, la philosophie se transforme en une sorte de chasse aux traces. Mais où sont-elles, les traces, étant donné qu'elles sont ce qu'il y a de moins visible? On l'a dit: à l'endroit où il y a le moins de présence. Il faudra donc chercher du côté du presque non-être, et espérer qu'il y aura rencontre avec ce qui, par constitution, n'est pas fait pour être rencontré. Autant dire qu'on est obligé de miser sur l'improbable et l'inespéré14 $\ldots$

Il s'ensuit, entre autres, que la philosophie est tout, sauf une activité naturelle ${ }^{15}$. On ne saurait donc la pratiquer dans le droit fil de ce qu'on vit, en ayant comme déclinatoire ce qui se passe habituellement dans le monde. Mais s'il est impossible de philosopher en restant dans le cours des choses, il n'y a de pensée qu'à la faveur d'une posture plutôt inconfortable... Pour le dire de façon à la fois plus précise et plus radicale: cette possibilité insolite ne se manifeste que si on arrive à convertir toute réalité dans une outrance par défaut ou par excès (étant entendu que le minime aussi peut comporter un surplus intéressant). Il faut entraîner en conséquence ce qui s'offre dans un mouvement d'hyperbole, chose qui déconcertera toujours les rationalistes étroits et les tenants du sens commun. En effet, poussées à leur limite, les choses perdent leurs propriétés et redeviennent d'une certaine manière informes ${ }^{16}$. D'où le caractère étrange de l'idée (elle contient éminemment ce qui la ruine), et au-delà, des problèmes philosophiques en général. Il s'avère qu'on ne peut les tailler à l'équerre, pour la simple raison que les problèmes trop cadrés, en philosophie, ne sont plus tels. De problèmes, ils deviennent thèmes, c'est-à-dire purs et simples contenus. On retombe alors dans le domaine du positif, lequel est régi par le principe du rendement, du bénéfice, de l'acquis, bref du résultat, avec toutes les menaces qui s'y associent pour la pensée, qui vont de l'expansion dominatrice à l'ankylose patrimoniale... Or la phénoménologie, singulièrement, s'efforce de dépasser cette aporie,

14. Héraclite, fr. 18 .

15. On sait qu'Aristote n'était pas de cet avis (voir la célèbre phrase inaugurale de la Métaphysique). Sur le caractère anti-naturel de la pensée, voir typiquement Frege, Ecrits logiques et philosophiques, trad. C. Imbert, Paris, Seuil, 1971, p. 180.

16. Et inhospitalières de surcroît, les maxima n'étant pas très accueillants... 
dans la mesure où elle conçoit la philosophie - pour faire vite - comme le monde moins le résultat, c'est-à-dire moins lui-même en tant que réalisé (ce qu'on pourrait appeler la déposition du Gestell). Car qu'est-ce que l'intentionnalité? Ramenée à sa plus simple expression, c'est la chose sur le point d'inexister, i.e. une certaine façon - très puissante en vérité - de suspendre le résultat. Il n'y a de pensée, en dernier ressort, que si on réussit à défalquer du monde l'idée d'achèvement et de gain - cette vieille obsession de $1^{\text {'Occident }}{ }^{17}$ ! C'est bien pourquoi la philosophie ne fait pas bon ménage avec la disposition dominante de notre époque, étant donné qu'elle ne peut exister qu'en quittant la sphère des pseudoévidences naturalistes (y compris économico-comptables...) ainsi que les conduites pragmatiques qui les secondent, et ce faisant les transforment en concrétions fatales. En droit, la philosophie ne peut même pas s'identifier avec la discipline du même nom - cette spécialité académique aux confins bien arrêtés et aux règles méthodologiques positivement définies. Vivant en toute rigueur dans l'élément de la soustraction infinie ${ }^{18}$, elle évolue dans une région où il n'y a plus ni racines ni repères. Mais alors, comment peut-elle garder un ancrage dans le monde?

$\grave{A}$ un tel niveau d'exigence, il sera toujours difficile d'asseoir quoi que ce soit (aussi bien sur une institution que sur une doctrine). Il n'y aura à tout prendre que des aperçus provisoires - échantillons en devenir, chiffres d'une recherche vouée à asymptote, toujours en deçà de la borne de l'adéquat. Qu'importe! Le philosopher vivant n'est qu'opération, et s'il arrive à suggérer un nouveau mode d'existence ${ }^{19}$, fût-il extraordinairement fragile, il aura tenu sa promesse. C'est que par le biais d'une procédure condensante, il aura réussi à enrichir le monde en l'appauvrissant. La philosophie est le monde advenu une deuxième fois - même et tout autre - dans le régime de l'idée.

17. C'est le coeur de la critique heideggerienne de la rationalité calculatrice techno-productiviste, qui se solde par l'éloge de la Gelassenheit.

18. Alain Badiou, Conditions, Paris, Seuil, 1992, p. 108-129 et 179-195.

19. Étienne Souriau, Les différents modes d'existence, Paris, PUF, 1943 (réédité en 2009). 